\title{
The study of human health effect induced by depth information of stereo vision film
}

\author{
Xiao Wang, Zhiyu Qian*, Lidong Xing, Shuai Jin, Baoyu Liu, \\ Zhongqiang Li and Jianhua Yin \\ Nanjing University of Aeronautics and Astronautics \\ Nanjing, P. R. China \\ *zhiyu@nuaa.edu.cn
}

Received 14 July 2014

Accepted 15 September 2014

Published 13 November 2014

\begin{abstract}
The stereo vision results from the interaction between geometrical optics and visual psychology. Large depth will bring discomforts for the results of ghosting and flicker. The relevance of the ratio of jumping out depth (RJD) and electroencephalogram (EEG) gravity frequency (GF) was explored to reflect human health under different three-dimensional (3D) depth information (mainly the negative disparity) displayed on a three-dimensional television (3D-TV) with shutter glasses. EEG was obtained from 10 volunteers when they were watching $3 \mathrm{D}$ film segments with different negative disparities. The brain GF map shows that the depth information has a stronger influence on the frontal lobe than on the occipital lobe. For regression analysis, nonlinear curve fittings of GF to RJD in Fp1, F3, O2 and T5 channels were mainly performed when RJD ranged from 0 to 3.4, while linear fittings were performed in some special RJD ranges. It also confirms that RJD above 2.2 may lead to discomfort to human body. Finally, it suggests a suitable RJD range for people to watch from the objective method. The outcomes can be used as a guidance to decrease human discomforts induced by 3D production.
\end{abstract}

Keywords: 3D-TV; negative disparity; human discomfort.

\section{Introduction}

Despite 3D (three-dimensional) technology becoming a historical change in modern industry that brings visual feast to people, its disadvantage especially the health impact is increasingly apparent in recent years. Some feel physical discomforts like eye strain, dizziness, even nausea after watching three-dimensional television (3D-TV).
Scientists are exploring the internal factors which can induce these discomforts by using subjective questionnaires or analyzing some physiological signals. For example, scientists have confirmed that watching 3D-TV for a long time may lead to visual fatigue. When watching time increases, the eye strain increases at the same time. ${ }^{1} \mathrm{Li}$ and his team certified that the event-related potential component

${ }^{*}$ Corresponding author.

This is an Open Access article published by World Scientific Publishing Company. It is distributed under the terms of the Creative Commons Attribution 3.0 (CC-BY) License. Further distribution of this work is permitted, provided the original work is properly cited. 


\section{Wang et al.}

P700 delayed as watching duration increased in 3D condition. ${ }^{2}$ Mun's experiment also showed that people with fatigue exhibit significantly reduced P600 amplitudes and delayed P600 latencies in the postviewing condition of one hour watching. ${ }^{3}$ In addition, the video contents, ${ }^{4}$ ambient illumination level, ${ }^{5}$ the screen size, ${ }^{1,6}$ the depth information of stereoscopic images $^{7,8}$ and the crosstalk ${ }^{9,10}$ also could cause discomfort. Moreover, the discomfort may relate to human physical conditions as well. Children are not fit for watching stereoscopic 3D viewing ${ }^{9,11}$ due to their susceptible vision. Some greater visual and motion sickness symptoms also occurred in 3D viewing. ${ }^{12,13}$ Based on these aspects, qualitative conclusions have been certificated in many aspects, however, quantitative analysis is still lacking.

In this paper, we are going to explore more detailed clues from changes in the electrophysiological signal parameters namely the EEG gravity frequency (GF) which varies with different depth information and hope to get the quantitative role of the 3D depth impact on human health.

\section{Materials and Methods}

\subsection{Depth information of 3D film}

Depth information is the key factor for the formation of stereoscopic effect in 3D films. Large depth may cause ghosting and flicker that will lead to discomfort among viewers watching 3D-TV. There are lots of parameters to represent depth information. The most common parameter is binocular disparity which is recognized as the most important factor to the depth perception ${ }^{14}$ in the medium visual distance (i.e., the visual distance is $10 \mathrm{~m}$ ). In our experiment, the visual distance is $3 \mathrm{~m}$, so we use convergence angle instead. ${ }^{14}$ As seen in Fig. 1, $\alpha$ is the convergence angle when people watch the object appearing on the screen. It can be represented by the visual distance $d$ and the pupil distance $\mathrm{AB}$ in Eq. (1). When the object is inside or outside the screen, the convergence angle $\gamma$ or $\beta$ will be used. The convergence angle $\gamma$ is equivalent to the positive disparity and the convergence angle $\beta$ is equivalent to the negative disparity.

$$
\alpha=2 \arctan \frac{\frac{A B}{2}}{d} .
$$

Since only the negative disparity is discussed in this paper, a new parameter called RJD (the ratio of

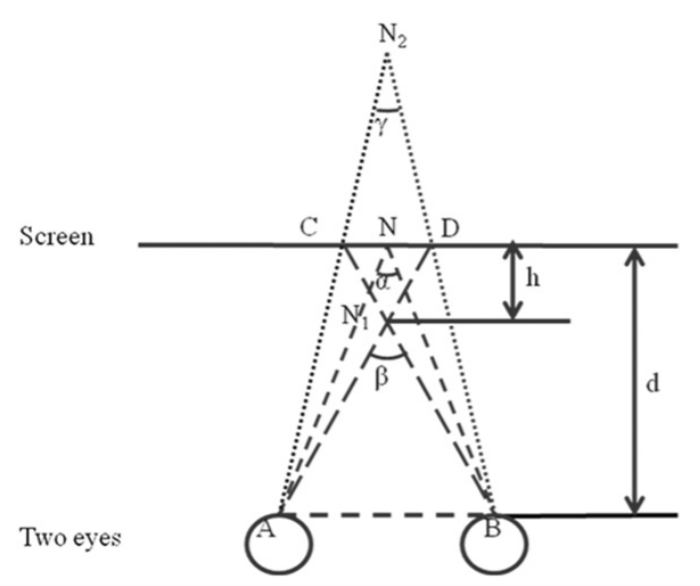

Fig. 1. Diagrammatic sketch of convergence angle and the RJD.

jumping out depth) was established to simplify the calculation complexity. In fact, the calculation of RJD is a part of the process in calculating the convergence angle. Equation (2) shows that RJD is a ratio which measures how far the object flys out of the screen. If RJD equals 0 , it means that the object is on the screen without any feeling of jumping out.

$$
\mathrm{RJD}=h / h_{s} .
$$

In Eq. (2), $h_{s}$ is the 3D-TV screen height. $h$ is the distance of the object jumping out of the screen. Since CD is the overlap distance of the left and the right picture which can be measured on the screen, $h$ will be elicited from the similar triangle theorem [Eqs. (3) and (4)].

$$
\begin{aligned}
h /(d-h) & =C D / A B, \\
h & =(C D \cdot d) /(A B+C D) .
\end{aligned}
$$

\subsection{Gravity frequency}

GF is an EEG factor that reflects the distribution of EEG power spectrum. ${ }^{15}$ It also represents the migration of EEG power spectrum in different conditions. ${ }^{16}$ It can be expressed as in Eq. (5), in which $\hat{p}(w)$ represents the power spectral density (PSD). $w$ represents the frequency of the EEG signal. $w_{2}$ and $w_{1}$ represent the top and bottom frequency which were respectively defined as $30 \mathrm{~Hz}$ and $0.5 \mathrm{~Hz}$ in this study.

$$
\mathrm{GF}=\frac{\sum_{w=w_{1}}^{w_{2}} \hat{p}(w) w}{\sum_{w=w_{1}}^{w_{2}} \hat{p}(w) w} .
$$




\section{Experiments}

\subsection{Volunteers}

Ten right-handed male volunteers (age: 22-25) participated in experiments. None was reported to have neurological disorders like epileptic seizures which would be an interference to EEG acquisition. All of them had normal stereo vision. They were requested to keep their scalp clean and have a good rest before the experiment was conducted. All volunteers were measured all over the experiment.

\subsection{Experiment film sources}

Film sources included four film segments which were cut from three 3D films named Open Season, Magic and Sky in the format of the left and right. They had different ranges of RJD. The minimum RJD was 0 and the maximum RJD was about 3.4 . The first and the second segments were major in small RJD while the third and the fourth segments were major in large RJD. The duration of each segment was $137 \mathrm{~s}$. RJD of each screenshot was calculated in s.

\subsection{Experiment procedure}

The experiments were conducted at $6: 30 \mathrm{pm}$, the room temperature was kept at $23.2 \pm 3.2^{\circ} \mathrm{C}$. Curtains were closed against the light disturbances. All film sources were displayed on a 3D-TV (Hisense, LED46XT39G3D). Volunteers wore a pair of shutter glasses (FPS3D02) while watching these segments. Four segments were played in order and all of them were in silent mode so that the interferences from the sound could be eliminated. At the beginning of each segment and at the end of the last segment, a 60-s relaxation was arranged for the volunteer. EEG signals were recorded throughout the experiment by a 16-lead electroencephalograph from Vishee (VEEG1320 Electroencephalograph).

\subsection{Parameter settings}

The electroencephalograph was set as $256 \mathrm{~Hz}$ sampling rate, $50 \mathrm{~Hz}$ notch and $0.5-30 \mathrm{~Hz}$ band-pass filtering. The electrode positions were a subset of the international 10-20 system sites (Fp1, Fp2, F3, F4, C3, C4, P3, P4, O1, O2, F7, F8, T3, T4, T5 and T6). The GND electrode was located between Fp1 and $\mathrm{Fp} 2$ on the forehead. A1 and A2 were two reference electrodes on each earlobe. All electrodes were referenced to the ground and later referenced to the mean between A1 and A2 digitally. The impedance level was kept under $30 \mathrm{~K}$ ohms until the experiment began.

\section{Results}

The EEG data of watching four film segments were analyzed. After excluding the artifacts like eyemovements and bad-blocks by EEGLAB, the GF was calculated in seconds. GFs of four segments were put together based on RJD, that is, GFs of 16 channels were integrated in accordance with RJD and GFs belong to the same channel and the same RJD would be set in one column. As a result, there were 16 columns totally. Each column was averaged after the integration and the GF mean value would be ranked in RJD ascending order ultimately.

Figure 2 shows the distribution of GF of 16 channels on the scalp when RJD increased. In order to have a constant RJD interval in the following regression analysis, we set one-fifth as an RJD step. This figure shows the total distribution of GF with different RJDs. It indicated that whatever the RJD was, GF increased from the forehead (Fp1, Fp2) to the occipital lobe (O1, O2, Oz).

Figure 3 shows some best curve fittings of GF to RJD in Fp1, F3, O2 and T5 channels, in which the $x$-axis represented the variation of RJD and the $y$-axis represented GF. Except that O2 channel had a linear decrease when RJD increased, Fp1, F3 and $\mathrm{T} 5$ channels had nonlinear curve fittings, especially in the majority of quadratic and cubic curves. The best curve fitting expressions of $\mathrm{Fp} 1(p=0.020$, $\left.R^{2}=0.495\right), \quad$ F3 $\quad\left(p=0.028, \quad R^{2}=0.467\right), \quad$ O2 $\left(p=0.013, \quad R^{2}=0.327\right) \quad$ and T5 $\quad(p=0.017$, $\left.R^{2}=0.419\right)$ channels were exhibited from Eqs. (6) to (9), and respectively $x$ represented RJD, $y$ represented GF.

$$
\begin{aligned}
& y=5.795-0.508 x+0.483 x^{2}-0.092 x^{3}, \\
& y=5.923-0.346 x+0.304 x^{2}-0.062 x^{3}, \\
& y=6.050-0.73 x, \\
& y=6.205+0.183 x-0.064 x^{2} .
\end{aligned}
$$

From the tendency of GF in Fig. 3, it was easy to find that some channels had an approximate linear change when RJD was divided into several subranges. Meaningful curve fittings of picked channels 
X. Wang et al.
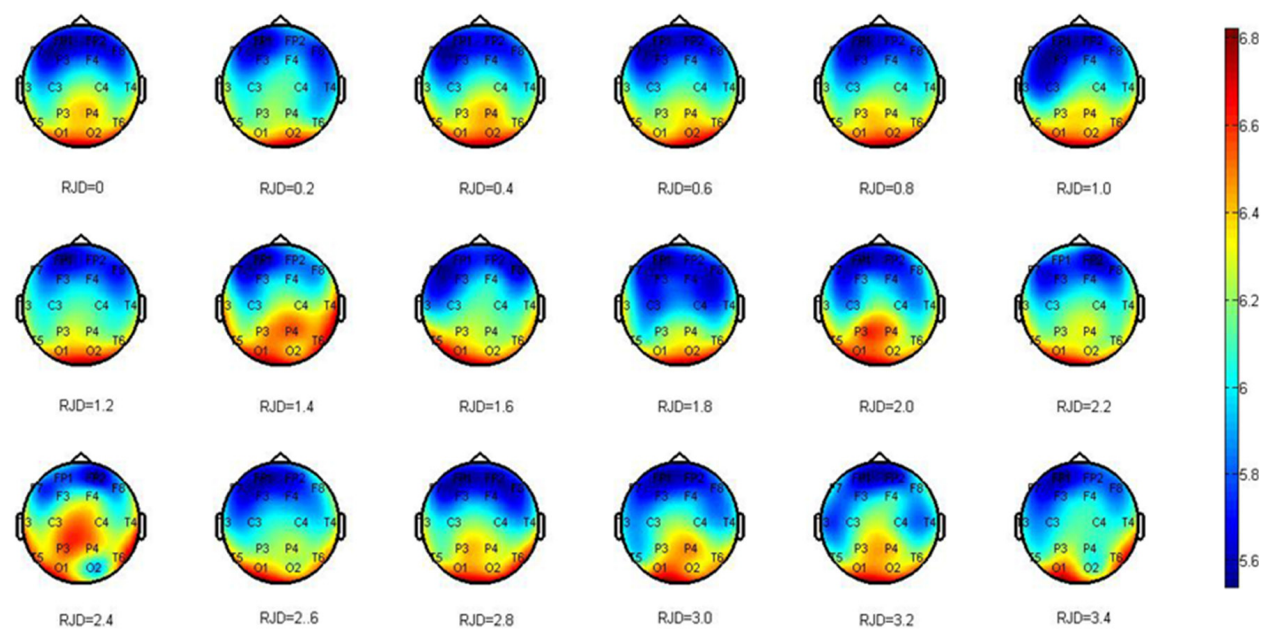

Fig. 2. The distribution of GF in different channels on the scalp. The color bar referred to the range of GF.
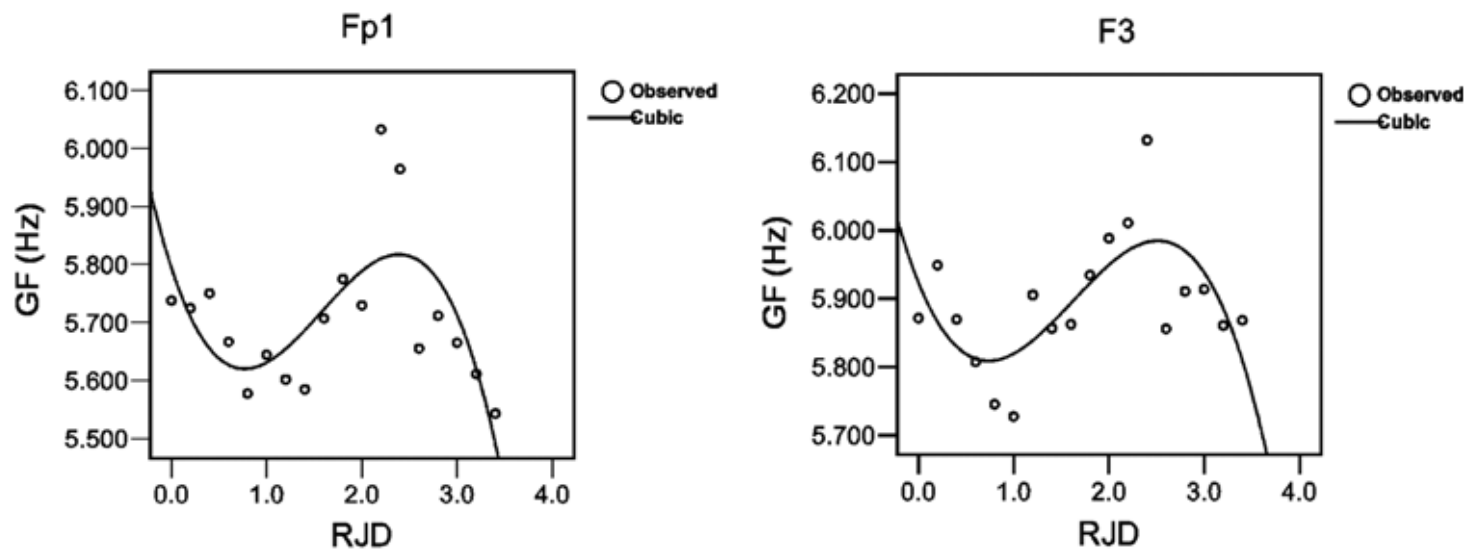

(a)

(b)

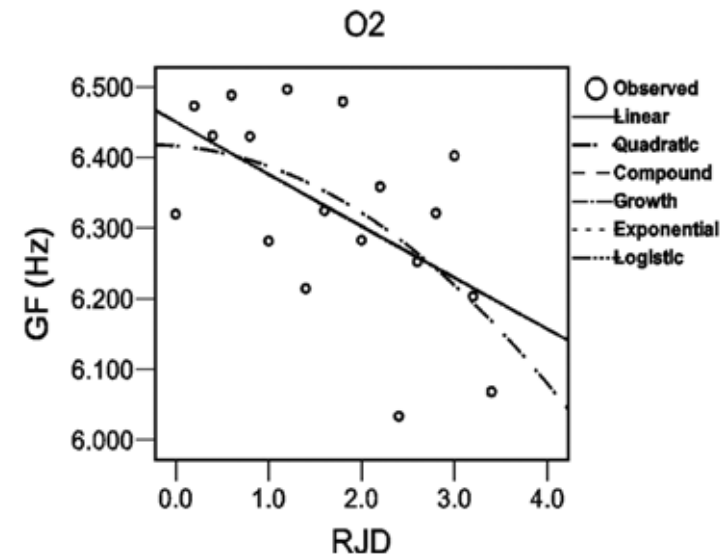

(c)

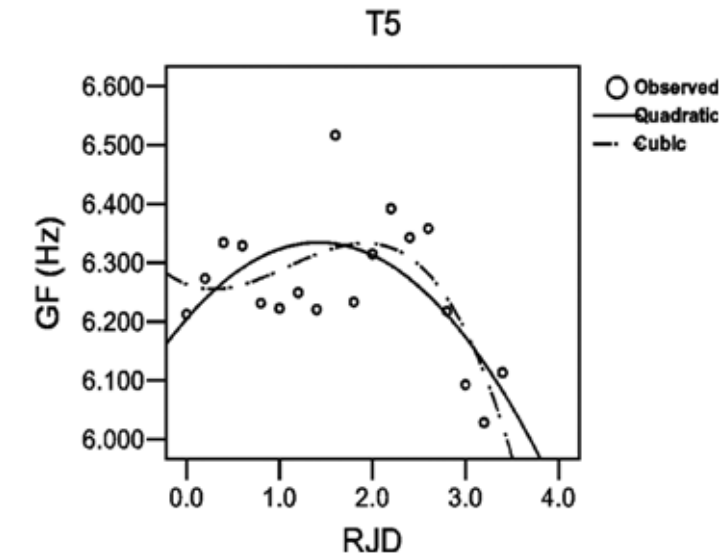

(d)

Fig. 3. The curve fitting of GF to RJD in different channels of (a) Fp1, (b) F3, (c) O2, (d) T5. The $x$-axis represents the variation of RJD and the $y$-axis represents GF. 
are drawn in Fig. 4, where $x$-axis and $y$-axis have the same meaning as in Fig. 3. As depicted in Fig. 4(a), if RJD was from 2.2 to 3.4, the change of GF was linear as well whereas some sub-ranges (i.e., RJD $=0-0.8)$ would have no meaningful curve fittings. Although some channels like Fp2 channel and C3 channel did not show well curve fittings in the total RJD range (RJD $=0-3.4$ ), the linear fittings in a certain sub-ranges were meaningful. The fitting equations of these channels were declared as follows:

$$
\begin{aligned}
& \text { Fp1: } \quad \text { RJD }=0-0.8 \quad \text { No curve fittings. } \\
& \mathrm{RJD}=0.8-2.2 \quad y=5.327+0.253 x \quad(p=0.012, \\
& \left.R^{2}=0.682\right) \\
& \mathrm{RJD}=2.2-3.4 \quad y=6.824-0.387 x \quad(p=0.005,
\end{aligned}
$$

Fp2: $\quad$ RJD $=0-0.6 \quad$ No curve fittings.

$$
\begin{aligned}
& \mathrm{RJD}=0.6-1.8 \quad y=5.569+0.154 x \quad(p=0.033, \\
& \mathrm{RJD}=1.8-3.4 \quad y=6.071-0.111 x \quad \begin{array}{l}
\left.R^{2}=0.630\right) \\
(p=0.007
\end{array} \\
& (p=0.020 \text {, } \\
& \left.R^{2}=0.779\right) \\
& \mathrm{RJD}=1.0-2.4 \quad y=5.540+0.228 x \quad(p=0.010 \text {, } \\
& \mathrm{RJD}=0-1.0 \quad y=6.122-0.246 x \quad(p=0.025 \text {, } \\
& \left.R^{2}=0.756\right) \\
& \mathrm{RJD}=1.0-2.4 \quad y=5.650+0.227 x \quad(p=0.040, \\
& \text { RJD }=2.4-3.4 \quad \text { No curve fittings. } \\
& \text { T5: } \quad \mathrm{RJD}=0-0.4 \quad y=6.212+0.304 x \quad(p=0.002 \text {, } \\
& \text { RJD }=0.4-2.2 \quad \text { No curve fittings. } \\
& \mathrm{RJD}=2.2-3.4 \quad y=7.085-0.309 x \quad(p=0.004, \\
& \left.R^{2}=0.835\right)
\end{aligned}
$$

\section{Fp1}

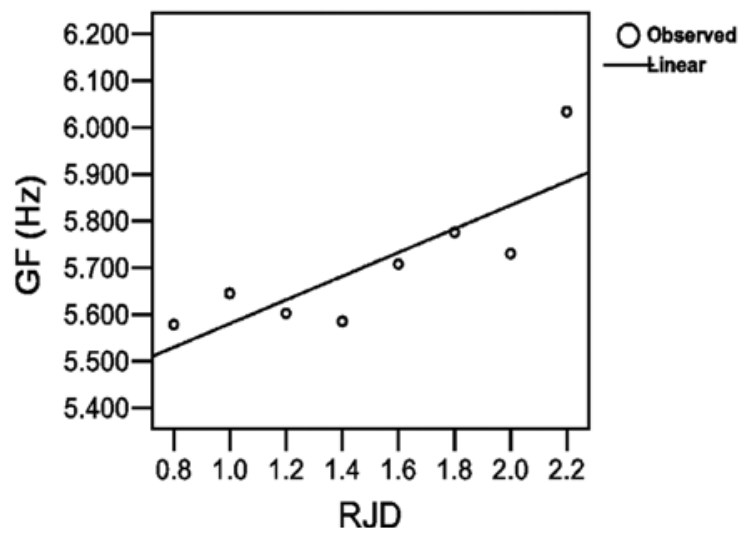

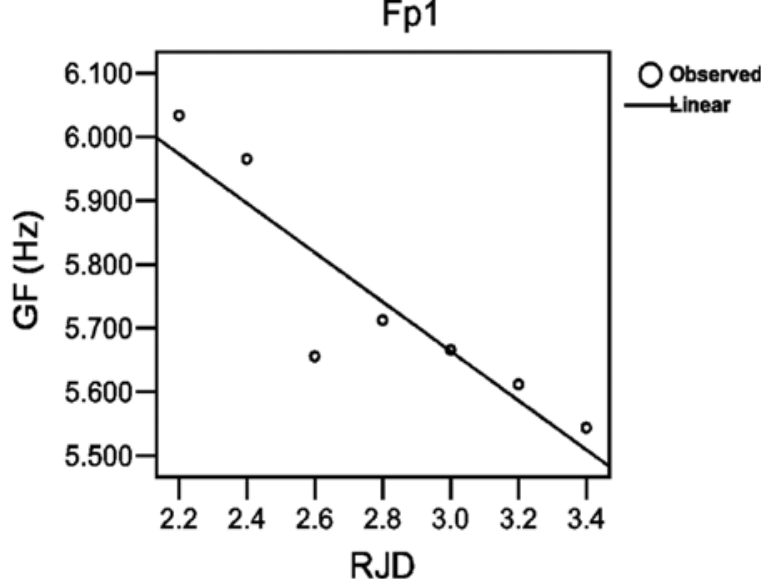

(a)
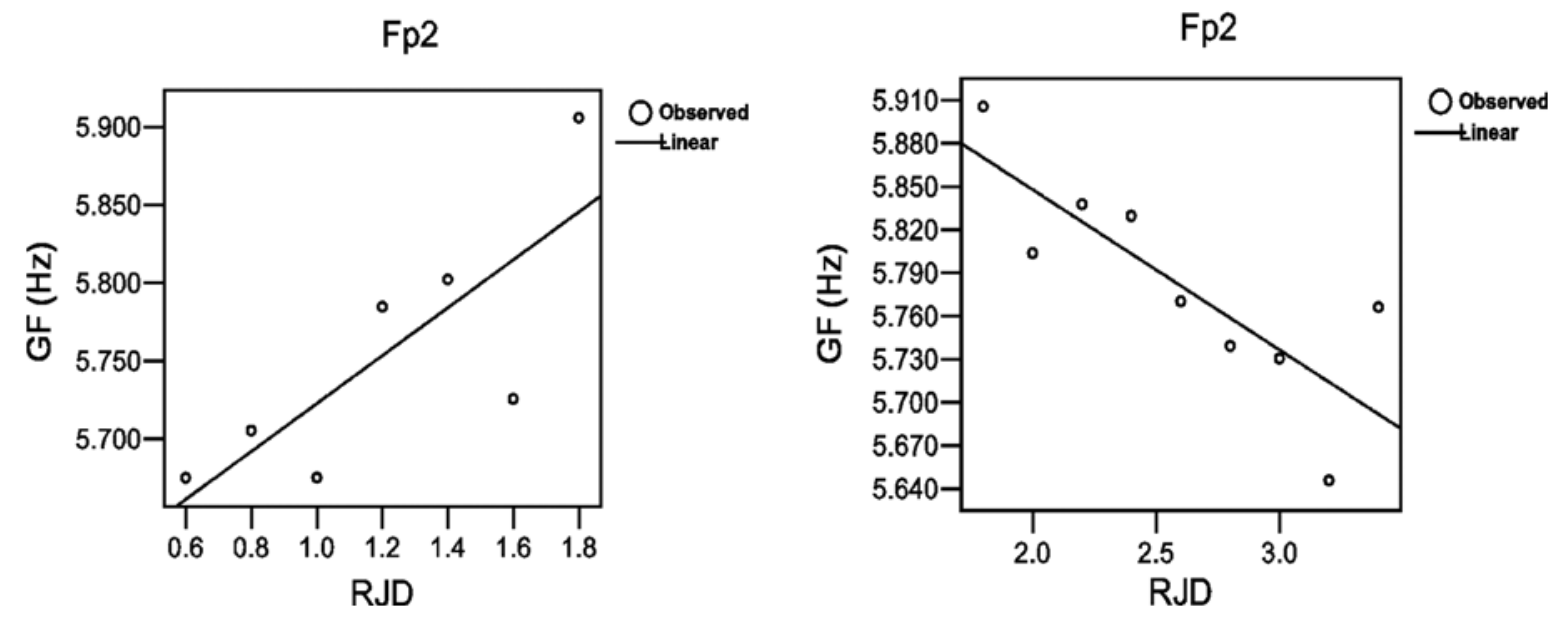

(b)

Fig. 4. Fragment curve fitting of GF to RJD in different channels of (a) Fp1, (b) Fp2, (c) F3, (d) C3, (e) T5. The $x$-axis represents the variation of RJD and the $y$-axis represents GF. 
X. Wang et al.

F3

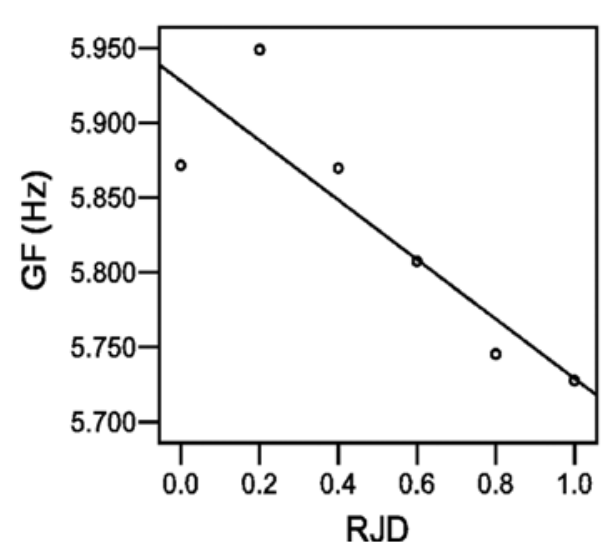

F3

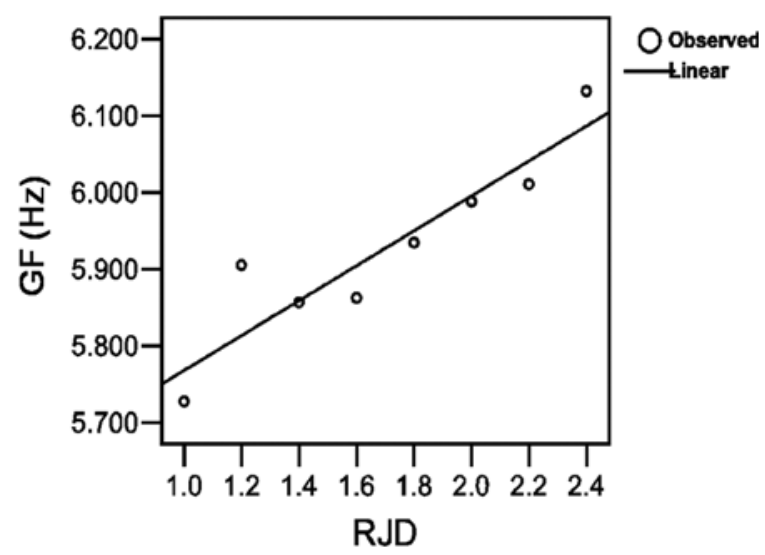

(c)

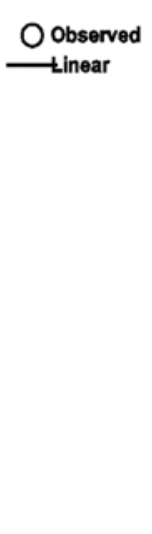

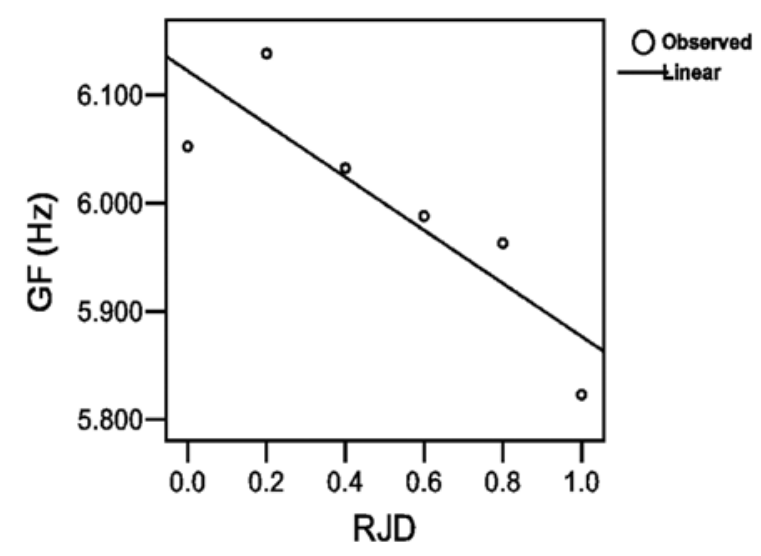

C3

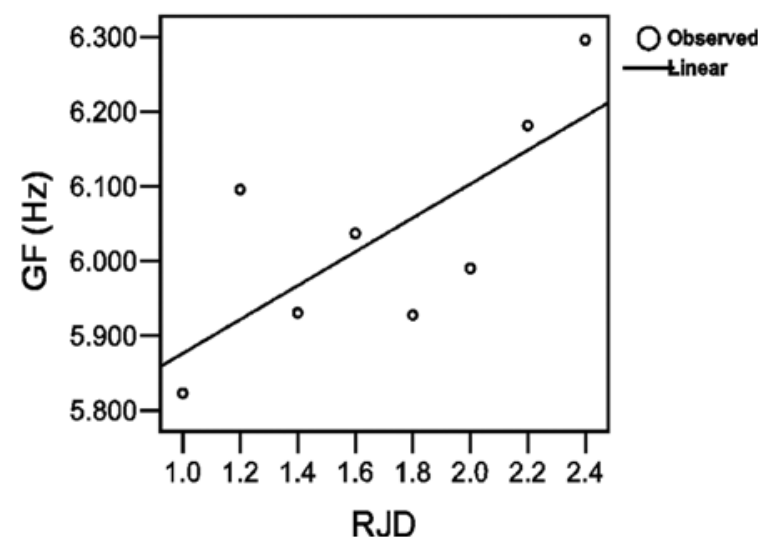

(d)
T5

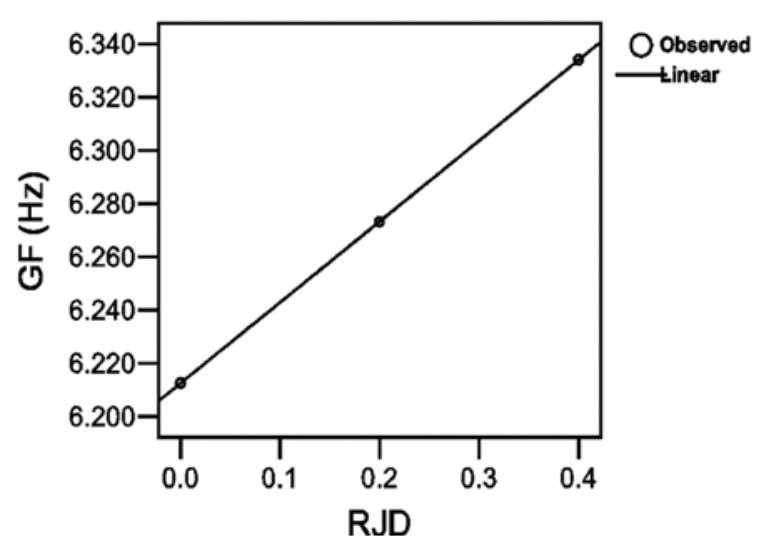

T5

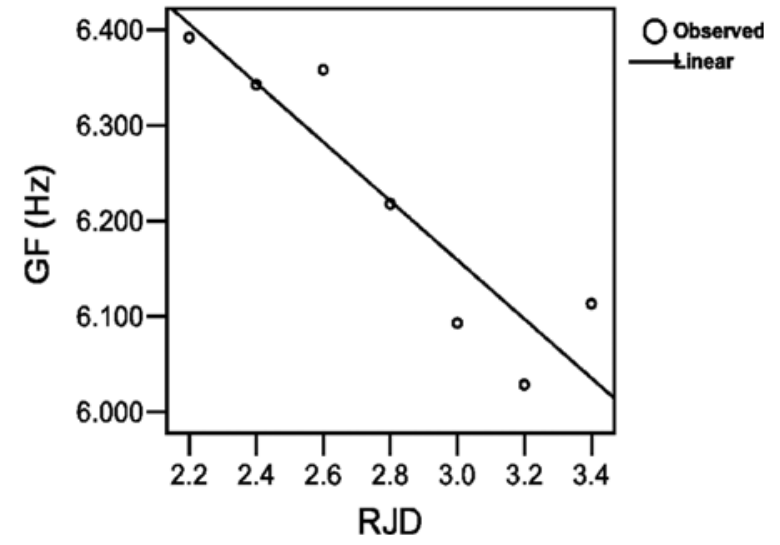

(e)

Fig. 4. (Continued) 


\section{Discussion}

Subjective questionnaires were also conducted. From the subjective questionnaires, few people had fatigue when watching small RJD 3D segments. However, most of them had uncomfortable symptoms of eyes when watching large RJD 3D segments. Their eyes felt dry and puffy. In addition, dizziness also emerged. It indicates that the depth information truly has an influence on human beings. Because of lack of researches on the cause of discomfort in 3D-TV, a simple, convenient and reliable quantitative measurement is necessary.

Since it has been certified that GF will shift to a low frequency when people have fatigue, GF seems to be a good parameter to measure the influence that depth information has on human body. When the fatigue increases, the cerebral cortex is inhibited more easily. ${ }^{17}$ Another study reported that GF could reflect the change of the emotion, for example, people who watched a sad movie would make GF become lower. ${ }^{15}$ In addition, it is known that some stimuli like imagination or perception activity will cause $\theta$ rhythm. According to this statement, RJD, as a visual stimulus in this paper, may create more $\theta$ rhythm and inhibit the high-frequency components. As shown in Fig. 2, GF on the scalp was in the range of $\theta$ rhythm, which was highest in occipital lobe and lowest in frontal lobe. It might be confirmed that the $3 \mathrm{D}$ effect had a stronger influence on the frontal lobe than on the occipital lobe. Combined with the subjective questionnaires, it might be also be explained why volunteers had discomfort in their eyes. Based on the curve fittings, it was easy to detect that $\mathrm{O} 2$ channel shows a linear decrease when RJD varied from 0 to 3.4. Theoretically the higher coefficient of determination $\left(R^{2}\right)$ seems to be better, but in some biostatistics papers, $\mathrm{R}^{2}$ usually can be accepted between 0.3 and $0.5 .^{18,19} R^{2}$ depends on the number of sample points, ${ }^{19}$ so the following result shows that $R^{2}$ improved when GF is expressed as an approximate linear role in a certain range of RJD. There were no meaningful fitting expressions in Fp1 and Fp2 when the RJD was between 0 and 0.6 (maybe it could be expanded to 0.8 ). It may indicate that discomfort is not obvious in this range which means the RJD in this range causes little effect to human bodies. Subtle changes in this range could be observed through F3, C3 and T5. When RJD was in the range 0.8 to 2.2 , all meaningful curve fittings of GF were raising. It is suggested that people could be in an excitatory state when seeing negative disparity in that RJD range. The above shows a suitable RJD range in $3 \mathrm{D}$ films for people to watch. However, when RJD was above 2.2, all meaningful curve fittings of GF were decreasing. It means that excessive negative disparity could lead to discomfort to human body. This was consistent with the ophthalmic research of eye discomfort when the negative disparity is more than $1^{\circ} .9,20$ According to the conversion between RJD and the negative disparity, it is easy to find that $\mathrm{RJD}=2.2$ is approximately equal to $1^{\circ}$ negative disparity. Therefore, $3 \mathrm{D}$ effect in this range is not suitable to appear frequently in 3D films.

There were also some limitations in this study. Only the volunteers' symptoms were collected, so the quantitative analysis of subjective evaluation to RJD could not be done. In other aspects, volunteers were undergraduate or graduate students in our lab. All of them were male students and similar age. Therefore, the limitation existed all at once. Regardless of the difference in pupil distance, we acquiesced in the pupil distance as a default of $65 \mathrm{~mm}$. But literatures reported that all points mentioned above would have a slight influence on the result. ${ }^{9,21,, 23}$

RJD is a more important new parameter, which simplifies the complexity of the calculation. Its principle is understandable and its value can be obtained manually. Actually it is a simple, convenient and reliable quantitative measurement to evaluate the influence of the 3D film depth information on human beings.

\section{Conclusion}

A simple and convenient parameter named RJD was defined in this study. It simplifies the computation complexity of the whole experiment. Owing to RJD, some detailed information comes out when the negative disparity changes. It is noticed that the depth information has a stronger influence on the frontal lobe than on the occipital lobe for the lowest GF in Fp1 channel and Fp2 channel. For regression analysis, the fitting equations of GF and RJD were deduced from EEG experiment. The equations are nonlinear in Fp1, F3 and T5, while the equation is linear in $\mathrm{O} 2$ when RJD ranges from 0 to 3.4. When RJD is divided into several ranges, the nonlinear role becomes linear in different ranges. Due to the 
discomfort which mainly occurs in eyes, the GF variation tendency of Fp1 and Fp2 channel seems to be more important. This paper also provides a suitable negative disparity range for people to watch flims in 3D and makes subjective responses more reliable. These quantitative results will help people decrease discomfort and maintain a healthy state. It provides further guidance for the $3 \mathrm{D}$ industry.

\section{Acknowledgment}

This research was supported by the Nature Science Foundation of China (NSFC) (61171059 \& 61075107), the twelfth "Five-Year Plan" National Key Technology R\&D Program (2012BAI23B07), Funding of Jiangsu Innovation Program for Graduate Education (KYLX_0248) and the Fundamental Research Funds for the Central Universities.

\section{References}

1. S. H. Cho, H. B. Kang, "The measurement of eyestrain caused from diverse binocular disparities, viewing time and display sizes in watching stereoscopic 3D content," 2012 IEEE Computer Society Conf Computer Vision and Pattern Recognition Workshops (CVPRW), pp. 23-28 (2012).

2. H. C. O. Li, J. Seo, K. Kham, S. Lee, "Measurement of $3 \mathrm{D}$ visual fatigue using event-related potential (ERP): 3D oddball paradigm," 2008 IEEE on 3DTV Conf. The True Vision-Capture, Transmission and Display of 3D Video, pp. 213-216 (2008).

3. S. Mun, M. C. Park, S. Park, M. Whang, "SSVEP and ERP measurement of cognitive fatigue caused by stereoscopic 3D," Neurosci. Lett. 525(2), 89-94 (2012).

4. K. Sakamoto, S. Asahara, S. Sakashita, K. Yamashita, A. Okada, "Influence of 3DTV video contents on physiological and psychological measurements of emotional state," 2012 IEEE 16th Int. Symp. Consumer Electronics (ISCE), pp. 1-4 (2012).

5. M. Pölönen, M. Salmimaa, J. Häkkinen, "Effect of ambient illumination level on perceived autostereoscopic display quality and depth perception," Displays 32(3), 135-141 ((2011).

6. A. Norkin, I. Girdzijauskas, "3DTV: One stream for different screens: Keeping perceived scene proportions by adjusting camera parameters," Picture Coding Symp. (PCS), pp. 489-492 (2012).

7. S. Yamamoto, S. Matsuoka, S. Yano, "The relationship between human mental variation and stereoscopic images - EEG approach," $A d v$. Human Factors/Ergon. 20, 827-832 (1995).

8. M. Huan, A. Minazuki, H. Hayashi, "Study on 3D image assessment using motion capture system," 2012 IIAI Int. Conf. Advanced Applied Informatics (IIAIAAI), pp. 182-186 (2012).

9. M. T. M. Lambooij, W. A. IJsselsteijn, I. Heynderickx, "Visual discomfort in stereoscopic displays: A review," Journal of Imaging Science and Technology, 53(3), 30201-1-30201-14 (2009).

10. M. T. M. Lambooij, W. A. IJsselsteijn, I. Heynderickx, "Visual discomfort of 3D TV: Assessment methods and modeling," Displays 32(4), 209-218 (2011).

11. W. Xiao, W. Fei, "A study of stereoscopic display technology and visual fatigue caused by viewing stereoscopic images," 2011 IEEE 2nd Int. Conf. Computing, Control and Industrial Engineering (CCIE) (2011), pp. 379-382.

12. D. Zhu, Q. M. Luo, G. M. Zhu, W. Liu, "Kinetic thermal response and damage in laser coagulation of tissue," Laser Surg. Med. 31, 313-321 (2002).

13. S. Yang, T. Schlieski, B. Selmins, S. C. Cooper, R. A. Doherty, P. J. Corriveau, J E Sheedy, "Stereoscopic viewing and reported perceived immersion and symptoms," Optometry Vis. Sci. 89(7), 1068-1080 (2012).

14. G. Y. Ying, "Flat 3D-TV and human eye psychology", Adv. Display. 10, 5-12 (2012).

15. Y. Lu, H. Wu, J. Qian, F. Xie, "Research on the influence of mozart sonata K.448 on EEG power spectrum and gravity frequency," Acta Biophys. Sin. 27(2), 154-166 (2011).

16. W. Klimesch, "EEG alpha and theta oscillations reflect cognitive and memory performance: A review and analysis," Brain Res. Rev. 29(2), 169-195 (1999).

17. C. Zhang, C. X. Zheng, Y. Ouyang, X. L. Yu, "Analysis of mental fatigue basing on power spectrum features of EEG," Space Med. Med. Eng. 21(1), 35-39 (2008).

18. Y. H. Chan, "Biostatistics 201: Linear regression analysis," Singapore Med. J. 45(2), 55-61 (2004).

19. A. C. Cameron, F. A. G. Windmeijer, "R-squared measures for count data regression models with applications to health-care utilization," J. Business Economic Stat. 14(2), 209-220 (1996).

20. M. Wöpking, "Viewing comfort with stereoscopic pictures: An experimental study on the subjective effects of disparity magnitude and depth of focus," J. Soc. Information Display 3(3), 101-103 (1995).

21. W. A. IJsselsteijn, P. J. H. Seuntiëns, L. M. J. Meesters, "State-of-the-art in human factors and 
quality issues of stereoscopic broadcast television," ATTEST (2002), pp. 1-41.

22. Y. Nakamura, "Measurement of pupillary unrest in eyestrain," Japanese J. Ophthalmol.40(4), 533 (1996).
23. K. Ukai, P. A. Howarth, "Visual fatigue caused by viewing stereoscopic motion images: Background, theories, and observations," Displays 29(2), 106116 (2008). 